\title{
Decoupling the Influence of Systemic Variables in the Peripheral and Cerebral Haemodynamics during ECMO Procedure by Means of Oblique and Orthogonal Subspace Projections*
}

\author{
A. Caicedo, Member, IEEE, I. Tachtsidis, M.D. Papademetriou, S. Van Huffel Fellow, IEEE
}

\begin{abstract}
Extra-Corporeal Membrane Oxygenation (ECMO) is a life support system for infants and children with cardio-respiratory failure. During ECMO it is possible to have unstable cerebral haemodynamics, due to strong oscillations in the systemic variables, among other factors, which may lead to brain damage in the patients. Therefore, monitoring the coupling between cerebral haemodynamics and systemic signals might alert us of possible imminent brain damage. In this study we explore the use of orthogonal and oblique subspace projections in the decoupling of these variables, by assessing the ratio between the projections of the haemodynamic variables, onto the subspace spanned by the systemic variables, and the original signals. The coupling of these two systems may differ as different protection mechanisms protect the peripheral system and the brain. Subspace projection was able to decompose the heamodynamic variables as a sum of components related to each systemic variable, separately. As expected, stronger coupling was found between the peripheral haemodynamic and the systemic variables.
\end{abstract}

\section{INTRODUCTION}

Extra-Corporeal Membrane Oxygenation (ECMO) procedure is a technique used to provide support for the heart and/or the lungs in patients with cardiac and/or respiratory failure. In veno-arterial (VA) ECMO patients are cannulated from

*This research was supported by: Research Council KUL: GOA MaNet, $\mathrm{CoE}$ EF/05/006 Optimization in Engineering (OPTEC), PFV/10/002 (OPTEC), IDO 08/013 Autism, several $\mathrm{PhD} /$ postdoc \& fellow grants; Flemish Government:

FWO: $\mathrm{PhD} /$ postdoc grants, projects: FWO G.0302.07 (SVM), G.0341.07 (Data fusion), G.0427.10N (Integrated EEG-fMRI), G.0108.11 (Compressed Sensing) G.0869.12N (Tumor imaging) research communities (ICCoS, ANMMM); IWT: TBM070713-Accelero, TBM070706-IOTA3, TBM080658-MRI (EEG-fMRI), PhD Grants; IBBT

Belgian Federal Science Policy Office: IUAP P6/04 (DYSCO, `Dynamical systems, control and optimization', 2007-2011); ESA AO-PGPF-01, PRODEX (CardioControl) C4000103224. EU: RECAP 209G within INTERREG IVB NWE programme, EU HIP Trial FP7-HEALTH/ 2007$2013\left(n^{\circ}\right.$ 260777) (Neuromath (COST-BM0601) Other: BIR\&D Smart Care.

A. C. is with the Electronic Engineering Department, ESAT/SCD SISTA, Katholieke Universiteit Leuven, Leuven, Belgium. He is also with the IBBT Future Health Department, Katholieke Universiteit Leuven, Leuven, Belgium (phone: +32 16 321067; e-mail: alexander.caicedodorado@esat.kuleuven.be).

I. T. and M.D. P. are members of the Biomedical Optics Research Laboratory at the Medical Physics and Bioengineering Department, University College London, London, UK (e-mail: iliastac@medphys.ucl.ac.uk and mpapa@medphys.ucl.ac.uk, respectively).

S. VH. is with the Electronic Engineering Department, ESAT/SCD SISTA, Katholieke Universiteit Leuven, Leuven, Belgium. She is also with the IBBT Future Health Department, Katholieke Universiteit Leuven, Leuven, Belgium (e-mail: sabine.vanhuffel@esat.kuleuven.be). the major neck vessels - right common carotid artery (RCCA) and internal jugular vein (IJV). Blood drains from the patient and gets oxygenated externally by the ECMO circuit and returns back to the patient. VA ECMO bypasses both the heart and lungs and provides cardiac and respiratory support. Veno-venous (VV) ECMO bypasses only the lungs and in this case the patient has only one double lumen cannula inserted in the IJV. VV ECMO does not provide cardiac support. Patients undergoing ECMO often have periods of haemodynamics instability, hypoxia and/or hypercapnia; in addition, physiological changes caused by the ECMO procedure itself may alter the cerebral autoregulation mechanisms due to multi-factorial reasons such as heparinitazion, hemodilution and reduced arterial pulsatility [1]; in consequence ECMO patients have increased risk for brain injury. Several studies have described changes in the cerebral haemodynamics before, during and after ECMO procedure. Liem et al. reported that mean arterial blood pressure (MABP), arterial oxygen saturation $\left(\mathrm{SaO}_{2}\right)$, transcutaneous partial pressure of oxygen $\left.(\mathrm{tcpO})_{2}\right)$ and transcutaneous partial pressure of $\mathrm{CO}_{2}(\mathrm{tcpCO})$ were some of the variables that better explained changes in total haemoglobin (HbT). Ejike et al. reported no significant correlation between changes in ECMO flow and regional oxygen saturation $\left(\mathrm{rScO}_{2}\right)$ and a negative correlation between $\mathrm{rScO}_{2}$ and $\mathrm{pCO}_{2}$. Papademetriou et al. described oscillations in brain and peripheral haemodynamics during changes in ECMO flow, as measured with near-infrared spectroscopy (NIRS), and its use as a marker of cerebral autoregulation [3, 4]. Studies about the coupling between systemic and haemodynamic variables have been performed by means of regression models [2,,5]. Although these approaches seem to provide good results they can be highly affected by the presence of multi-collinear variables and nonstationarities. In a previous work [6] we aimed to study the interactions of these variables by means of Canonical Correlation Analysis (CCA). Although CCA provides a strong theoretical framework to study the coupling between multivariable datasets, the results are difficult to analyze which reduce its clinical value.

Subspace projections methods, on the other hand, address straightforwardly this problem producing results that are not only easy to interpret mathematically but also clinically. The main goal of subspace projection techniques in signal processing is to mitigate the influence of noise in a signal of interest. Thus, orthogonal subspace projection (OrSP) and oblique Subspace Projection (OSP) aim to reduce orthogonal 
and oblique noise, respectively; where the noise can be due to measurement noise or contamination by other signals dynamics. In this study we aim to decouple the dynamic of the systemic variables (SV) from the cerebral-peripheral haemodynamic variables (CHV, PHV) by means of OrSP and OSP in combination with the discrete wavelet decomposition of the SV. In addition the strength of the coupling was quantified using features extracted from the decoupled signals.

\section{METHODS}

\section{A. Data}

Measurements from a patient, 3 days old, undergoing ECMO procedure were used in this study. A dual channel near infrared spectroscopy (NIRS) system (NIRO 200, Hamamatsu Photonics KK) was used to measure changes in oxy- and deoxy- haemoglobin concentrations $\left(\mathrm{HbO}_{2}\right.$ and $\mathrm{HHb}$ ), and tissue oxygenation index (TOI), which is calculated as $\mathrm{HbO}_{2} /\left(\mathrm{HbO}_{2}+\mathrm{HHb}\right)$, using spatially resolved spectroscopy. NIRS data were collected at a frequency of $6 \mathrm{~Hz}$. Channel 1 was placed on the forehead in order to assess cerebral haemodynamics, while channel 2 was placed on the calf to assess peripheral haemodynamics. Systemic data: mean arterial blood pressure (MABP), end-tidal $\mathrm{CO}_{2}$ $\left(\mathrm{EtCO}_{2}\right)$, heart rate $(\mathrm{HR})$, core temperature (Core $\left.\mathrm{T}\right)$ and arterial oxygen saturation $\mathrm{SaO}_{2}$; were continuously measured in real time at the bedside (Intellvue MP70, Philips Medical Systems).
MABP was obtained invasively from a radial artery. $\mathrm{SaO}_{2}$ was obtained from a pulse oximeter placed on the toe of the patient. MABP was recorded at $125 \mathrm{~Hz}$ while $\mathrm{EtCO}_{2}$ was recorded at $62.5 \mathrm{~Hz}$. All data were downsampled to $1 \mathrm{~Hz}$ and artifacts were removed manually by using interpolation. In figure 1 the systemic and NIRS measurements from the patient are shown. The data was collected during changes in the ECMO flow. The ECMO flow was initially decreased from baseline level (100\%) in steps of $10 \%$ down to $70 \%$, and then it was increased, following the same profile, until baseline level was reached.

\section{B. Subspace Projections}

In linear algebra a projection onto a subspace can be performed by the use of a projection matrix. A projection matrix is then an operator that maps any vector in a subspace spanned by its column space. A general overview of subspace projections and its properties can be found in [7]. In subspace projection techniques the goal is to find a projection matrix that performs this task. Let the matrix $\boldsymbol{X}$ of size $m x n$ be composed of the vectors $\left\{\begin{array}{lll}x_{1} & x_{2}, \ldots x_{n}\end{array}\right\}$, with $x_{i} \in \mathfrak{H}^{n}$, in its columns. Assuming that $\boldsymbol{X}$ is full rank (rank = $n$, with $m>n$ ) the vectors $\left\{x_{1}, \ldots, x_{n}\right\}$ form a basis for the column space of $\boldsymbol{X}, \boldsymbol{C}(\boldsymbol{X})$. Given $\mathbf{X}$, the orthogonal projection matrix $\boldsymbol{P}=\boldsymbol{X}\left(\boldsymbol{X}^{T} \boldsymbol{X}\right)^{-1} \boldsymbol{X}^{T}$, is the matrix that projects any vector $y \in \mathfrak{R}^{m}$ in $\mathbf{C}(\mathbf{X})$. This projection is calculated as $y_{X}=\boldsymbol{P} y$. Whilst its projection in the orthogonal subspace of $C(\boldsymbol{X})$, namely $C\left(\boldsymbol{X}^{\perp}\right)$ or the null space of $\mathbf{X}^{\mathrm{T}} \mathrm{N}\left(\mathbf{X}^{\mathrm{T}}\right)$, is $y_{\boldsymbol{X}}{ }^{\perp}=(\boldsymbol{I}-\boldsymbol{P}) y$, with $y=y_{X}+y_{X} \stackrel{\perp}{\text {. }}$
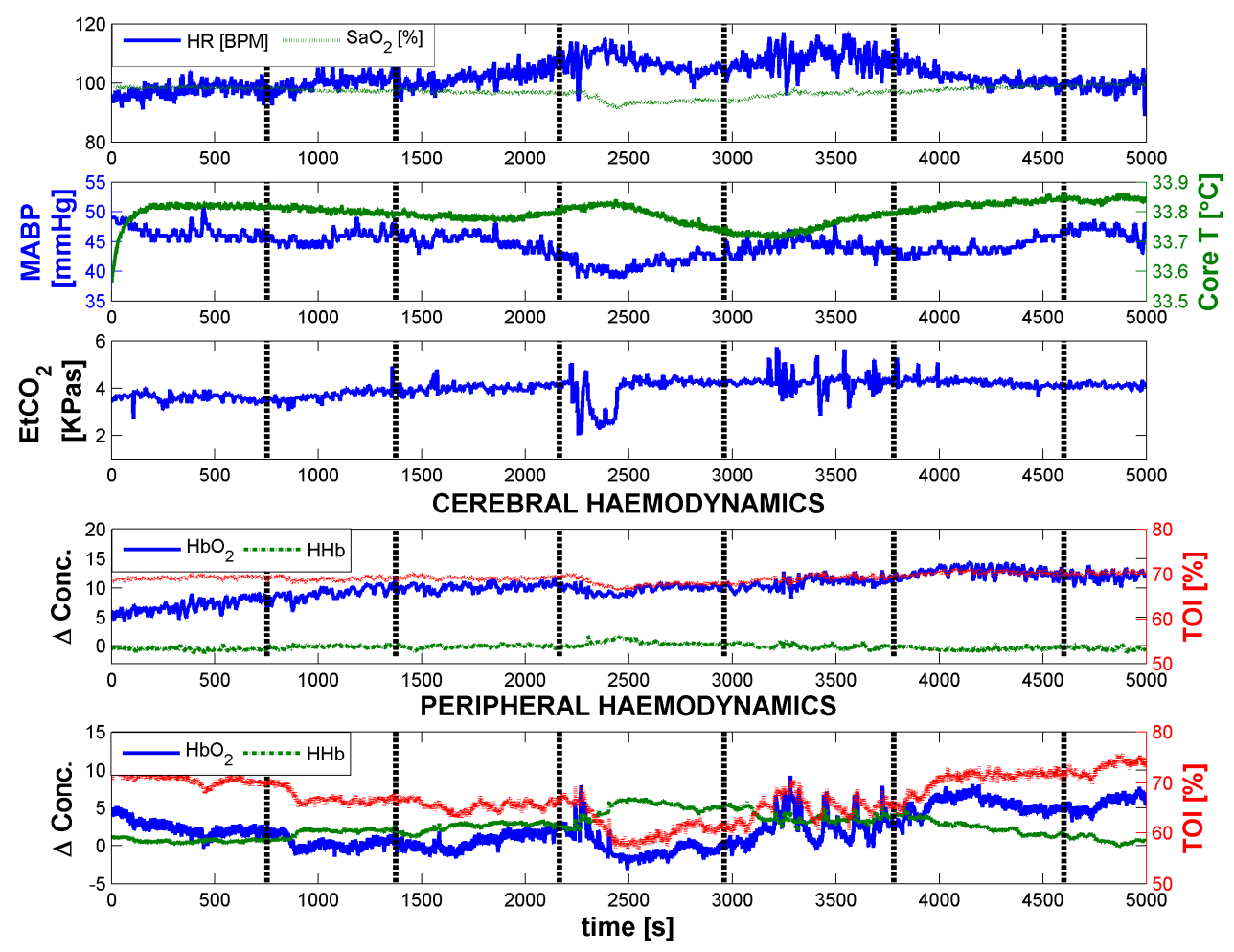

Figure 1. Systemic and Haemodynamic variables measured from the patient. The dotted vertical Lines represent the changes in the ECMO flow. The flow was first decreased from baseline level (100\%) up to $70 \%$ in steps of 10\%, afterwards it was returned to the baseline level following the same profile. 
An oblique projection, on the other hand, is a projection that is carried out along oblique subspaces. In the case of an orthogonal projection, the projection was performed along $C(\boldsymbol{X})$ and $N\left(\boldsymbol{X}^{T}\right)$. Those 2 subspaces where completely defined by the data in $\boldsymbol{X}$. However, for an oblique projection a second reference is needed, as the projection should be performed parallel to this reference subspace. Let a matrix $\mathbf{Z}$ be composed of linearly independent vectors $\left\{\begin{array}{lll}z_{1} & , z_{2}, . . & z_{p}\end{array}\right\}$, $z_{i} \in \mathscr{A}^{m}$, then the projection matrix that projects a vector $y$ in $C(\boldsymbol{Z})$ along $C(\boldsymbol{X})$, denoted by $\mathbf{E}_{\mathbf{Z X}}$, and the projection matrix that projects a vector y in $C(\boldsymbol{X})$ along $C(\boldsymbol{Z})$, denoted by $\mathbf{E}_{\mathbf{X Z}}$, are given by:

$$
\begin{aligned}
& E_{Z X}=\left[\begin{array}{ll}
Z & 0
\end{array}\right]\left[\begin{array}{cc}
Z^{T} Z & Z^{T} X \\
X^{T} Z & X^{T} X
\end{array}\right]\left[\begin{array}{l}
Z^{T} \\
X^{T}
\end{array}\right] \\
& E_{X Z}=\left[\begin{array}{ll}
0 & X
\end{array}\right]\left[\begin{array}{ll}
Z^{T} Z & Z^{T} X \\
X^{T} Z & X^{T} X
\end{array}\right]\left[\begin{array}{l}
Z^{T} \\
X^{T}
\end{array}\right]
\end{aligned}
$$

The geometrical interpretation of subspace projections can be seen in figure 2 .

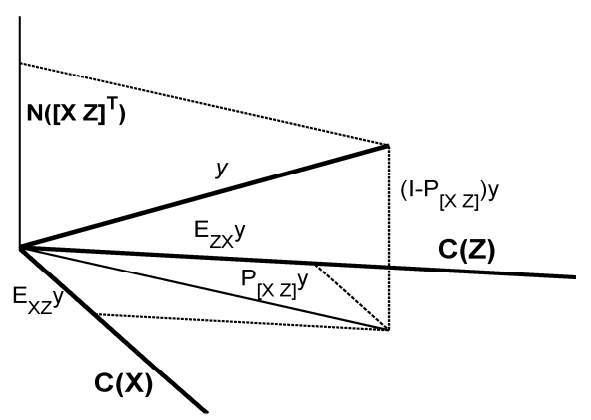

Figure 2. Geometrical interpretation for orthogonal and oblique projections.

\section{Decoupling Algorithm}

The problem was addressed in two different ways. On one hand, the projection of the cerebral and peripheral haemodynamic variables ( $\mathrm{CHV}, \mathrm{PHV}$ ) in the subspace spanned by the systemic variables (SV) decomposes $\mathrm{CHV}$ and $\mathrm{PHV}$ in a part that is linearly related to SV and a part that is orthogonal to it. The orthogonal part can be interpreted as the part of the haemodynamic variables (HV) that is related to measurement noise, not measured variables and nonlinear coupling with SV. On the other hand, the CHV and PHV can be projected in each one of the SV by calculating the corresponding oblique projection matrices; where the matrix $\boldsymbol{Z}$, in (1) and (2), corresponds to the signal where we would like to project the data and the matrix $\boldsymbol{X}$ to the remain SV. However, if raw signals are used to define $\mathrm{C}(\mathbf{X})$ the projection matrices will be highly sensitive to nonstationarities and high noise levels. Therefore, a change of the raw SV signals by the wavelet transform of the SV, as basis for $\mathrm{C}(\boldsymbol{X})$, is proposed. The use of the wavelet transform not only mitigates the effect of noise in the projections but also, intuitively, introduces the effects of non-stationaries. In this study we used 5th level discrete wavelet decomposition, with Daubechies 4 as wavelet mother. Moreover, in order to account for possible coupling delays, the wavelet coefficients of SV and its delayed versions were included in the matrix $\boldsymbol{X}$. In this study a delay of three samples was used. The use of the delays can be interpreted as imposing a model structure in the projection matrices. In addition, the ratios between the power of the projected and original signals, were computed as a measure of the coupling between the variables. In summary the algorithm is reduced to the following steps:

- Compute the wavelet transform of SV. Concatenate the coefficients and its delayed versions in a matrix $\boldsymbol{X}$ to form the basis for $\mathrm{C}(\boldsymbol{X})$.

- Compute the OrSP matrix and project the data. From this projection compute the ratio between the power of the projected signals and the power of the original signal.

- Compute the OSP matrix for each SV and project the data. From the projected signals compute the ratio between the projected variable and the original signal.

The different ratios will be used as a measure of the strength of the coupling between $\mathrm{CHV} / \mathrm{PHV}$ and SV, namely $\mathrm{P}_{\mathrm{C}}$ and $\mathrm{P}_{\mathrm{P}}$ respectively. The ratios can be interpreted as the amount of power in the CHV/PHV due to the SV in total, in case of an orthogonal projection, or separately, in case of oblique projection. The ratio $\mathrm{P}_{\mathrm{C}} / \mathrm{P}_{\mathrm{P}}$ can be used as a monitoring variable to assess the status of the mechanisms that regulates cerebral haemodynamics.

\section{RESULTS}

In figure 3, the decomposition of the cerebral and peripheral TOI in the components corresponding to each SV is shown. The peripheral TOI is more affected than the cerebral TOI by variations in SV; this might be due to intrinsic mechanisms that regulate cerebral haemodynamics. The ratio $\mathrm{P}_{\mathrm{C}} / \mathrm{P}_{\mathrm{P}}$ was 0.62 for $\mathrm{HbO}_{2}, 0.058$ for $\mathrm{HHb}$ and 0.045 for TOI. In table 2 the ratios between the powers of the projected $\mathrm{CHV} / \mathrm{PHV}$ in the $\mathrm{SV}$ are displayed. These ratios reveal the SV that affect at most the HV. This table shows that variations in $\mathrm{SaO}_{2}$ possess the highest impact in the variations of $\mathrm{CHV}$ and PHV. However, its influence is more pronounced in the peripheral haemodynamics. In addition $\mathrm{EtCO}_{2}$ appears to have a higher impact in the cerebral than in the peripheral haemodynamics.

\section{DISCUSSION}

OrSP and OSP were able to decompose the CHV/PHV in a series of components related to each SV. This approach is useful as it serves as a basis for the quantification of the coupling between the haemodynamics and the systemic variables. By calculating the ratios between the power of the components in the decomposed signal and the original signal the strength of the coupling was assessed. By including wavelets as a basis for the subspace of the systemic variables 
the non-stationary effects are taken into account; however, further studies are needed in order to validate these results and evaluate their clinical impact. In addition, the effect of nonlinear coupling should be further studied, as it is not included in the current analysis. In summary, in this study we have presented a way to decouple the influence of SV in $\mathrm{CHV}$ and PHV. This information may yield high clinical impact as it can alert the clinician which variable has the highest impact in the cerebral haemodynamics.

\section{REFERENCES}

[1] K. Liem, J. Hopman, B. Oesenburg, A. De Haan, C. Festen, and L. Kollee, "Cerebral Oxygenation and hemodynamics during induction of extracorporeal membrane oxygenation as investigated by near infrared spectrophotometry”. Pediatrics 93:555-561, 1995.

[2] J. Ejike, K. Schenkeman, K. Seidel, C. Ramamoothy, and J. Roberts. "Cerebral oxygenation in neonatal and pediatric patients during venoarterial extracorporeal life support". Pediatric Critical Care Medicine 7:154-158, 2006.
[3] M.D. Papademetriou, I. Tachtsidis, T.S. Leung, M.J. Elliott, A. Hoskote, C.E. Elwell. "Cerebral and Peripheral Tissue Oxygenation in Children Supported on ECMO for Cardio-Respiratory Failure". Adv.Exp.Med.Biol 662:447-53, 2010.

[4] M.D. Papademetriou, I. Tachtsidis, M. Banaji, M.J. Elliott, A. Hoskote, C.E. Elwell. "Optical topography to measure variations in regional cerebral oxygenation in an infant supported on veno-arterial extra-corporeal membrane oxygenation". Adv Exp Med Biol.. 737:716. 2012.

[5] M.M. Tisdal, C. Taylor, I. Tashtsidis, T.S. Leung, C.E. Elwell, and M. Smith. "The effect on Cerebral Tissue Oxygenation Index of Changes in the Concentrations of Inspired Oxygen and End-Tidal Carbon Dioxide in Healthy Adult Volunteers". Anesth Anal 109(3): 906-913, 2009.

[6] A. Caicedo, M.D. Papademetriou, C.E. Elwell, A. Hoskote, M.J. Elliott, S. Van Huffel, and I. Tachtsidis. "Canonical Correlation Analysis in the Study of Cerebral and Peripheral Haemodynamics Interrelations with systemic Variable sin Neonates Supported on ECMO”. Adv. Exp. Med. Biol. Waiting for publication.

[7] R.T. Behrens and L.L Scharf. "Signal Processing Applications of Oblique Projection Operators". IEEE Trans. Signal Process. 42(6) pp. 1413-1423, June, 1994.

Peripheral Haemodynamics

Cerebral Haemodynamics

\begin{tabular}{|l|c|c|c|c|c|c|c|c|c|c|c|c|}
\cline { 2 - 14 } \multicolumn{1}{c|}{} & $\mathrm{HR}$ & $\mathrm{MABP}$ & Core T & $\mathrm{SpO}_{2}$ & $\mathrm{EtCO}_{2}$ & Flow & $\mathrm{HR}$ & $\mathrm{MABP}$ & Core $\mathrm{T}$ & $\mathrm{SpO}_{2}$ & $\mathrm{EtCO}_{2}$ & Flow \\
\hline $\mathrm{HbO}_{2}$ & 2.23 & 15.13 & 1.68 & 38.20 & 4.02 & 5.57 & 5.43 & 2.07 & 4.24 & 26.63 & 17.89 & 8.83 \\
\hline $\mathrm{HHb}$ & 5.03 & 7.55 & 0.79 & 68.09 & 9.31 & 1.43 & 0.41 & 4.76 & 4.25 & 48.70 & 1.39 & 4.44 \\
\hline $\mathrm{TOI}$ & 0.49 & 7.28 & 0.56 & 70.57 & 0.37 & 9.63 & 1.72 & 2.41 & 0.32 & 71.61 & 4.63 & 0.46 \\
\hline
\end{tabular}

Table 1. Percentage of power in the corresponding Peripheral or Cerebral Haemodynamic variable (left-column) due to the systemic variables (second row).

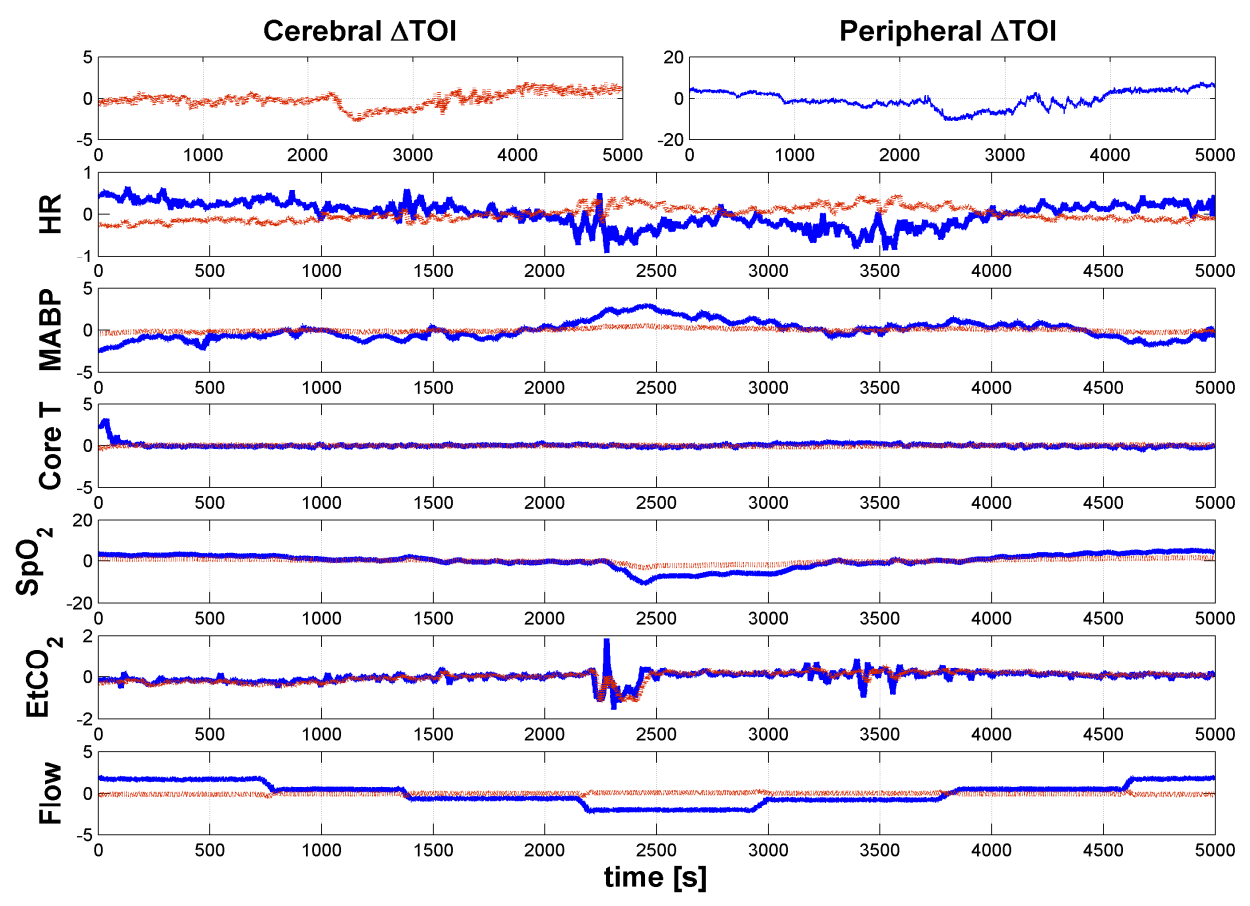

Figure 3. Decomposition of the Cerebral and Peripheral TOI in the components related to each one of the systemic variables under analysis. In red the decomposition for the cerebral TOI is shown, whilst blue represents the decomposition for peripheral TOI. 